\title{
ANALYSIS OF THE CURRENT SITUATION IN CARE PROVIDED IN EARLY AGE AND SPEECH THERAPY INTERVENTION IN INDIVIDUALS WITH AUTISTIC SPECTRUM DISORDER
}

\begin{abstract}
Alena Ř́hová
\section{Annotation}

This paper introduces the results of a research focused on the analysis of the current situation concerning the care provided in early age and speech therapy intervention in individuals with autistic spectrum disorder. The research was carried out between April and July 2010 in the Olomouc region and abroad (Slovak Republic - Bratislava). A questionnaire, the selected research method, was distributed to speech therapists, experts (specialists in education and in special education, psychologists, psychiatrists, paediatricians and neurologists) and parents of children with autistic spectrum disorder. The article provides an introduction to the studied problem, defines the research objectives, provides an analysis of the information received via the questionnaire and offers a conclusion.
\end{abstract}

\section{Key words}

Autistic spectrum disorders, early age, early care, speech therapy intervention.

\section{Introduction}

Autistic spectrum disorders (hereinafter also as "ASD”) are one of the most serious disorders of a child's mental development (Kurt-Lasocka, 1994; Thorová, 2006; Weber, 1970). The development of a child suffering from ASD is aberrant from infancy as the child is intact and it is deeply disturbed. Jelínková (1999) mentions that this category includes serious developmental disorders originating in early childhood and characterized by a quantitative disorder of social interaction, communication and an inclination towards stereotype and ritual behaviour. Therefore, it concerns a pervasive developmental disorder (Was ist autismus?, 2009). "The word pervasive means penetrating all and means that the development of a child is deeply disturbed in many respects” (Čadilová, Jůn, Thorová, 2007, p. 12). Autistic spectrum 
disorders primarily concern communication disorders. The disturbed communication ability is the principal symptom of the ADS clinical picture and belongs among the diagnostic triad mentioned above. The late development of speech is very often the primary reason of concern mentioned by parents (Gillberg in Thorová, 2006). Thorová (2006) discusses the fact that the communication ability deficit is to be found in reception, expression, in verbal and non-verbal communication and preverbal interaction skills. Peeters (1998) points out the fact that in about one half of the individuals with ASD verbal expression is absent and in $75 \%$ there is meaningful echolalia. In the works of Chakrabarti and Fombonn (2001) the prevalence mentioned is 40.0 and in the works of Bertrand et al. it is 67.0/ 10,000 (in Hrdlička, 2004). Hrdlička (2010) draws the attention to the radically changing degree of this value to $90 / 10,000$. Of course, we have to consider the possibility of positively false diagnoses. However, the necessity for numerous researches dedicated to this area remains a fact.

The research focuses on mapping the current situation in providing care in early care and speech therapy intervention in individuals with autistic spectrum disorder. It has been carried out in the Olomouc region and abroad (Slovak Republic). The lead researcher is Mgr. Alena Ríhová. The research team includes doc. Mgr. Kateřina Vitásková, Ph.D., Mgr. Lucia Pastieriková and Mgr. Eva Urbanovská. A questionnaire is the selected research method compiled applying the principle of combining the closed (structured) and open (non-structured) items. Furthermore, contact and filtration items are utilized. With respect to the contents, items ask for facts or knowledge or feelings, opinions and incentives. The questionnaire respects important requirements such as clarity, comprehensibility, avoidance of suggestive items, definiteness and primary focus on essential information.

In order to provide comprehensive and as efficient care for ADS clients as possible, the involvement of a number of experts and their mutual communication is necessary. Therefore, we distributed the questionnaire to speech therapists, specialists in education and special education, psychiatrists, psychologists, paediatricians and neurologists.

Parents play an indispensable role in the interdisciplinary team taking care of a person with ADS. Their proper and early education and psychotherapeutic support may represent the critical factor affecting the efficiency of the therapeutic and educational process. Thus the questionnaires were also distributed to parents with children suffering from ADS. 
Regarding the heterogeneity of respondents, three types of questionnaires were designed. First one for speech therapists, second one for experts (specialists in education and special education, psychologists, psychiatrists, paediatricians and neurologists) and a third one for parents with children suffering from ASD.

We set the following research objectives:

Main objectives:

1. Map out the current situation concerning care provided at an early age to individuals suffering from ASD;

2. Map out the current situation in speech therapy intervention in individuals suffering from ASD;

3. Compare the current situation concerning care provided at an early age to individuals with ASD in the Olomouc region and abroad (Slovak Republic);

4. Compare speech therapy intervention in individuals with ASD in the Olomouc region and abroad (Slovak Republic).

Partial objectives:

1. Learn about the awareness of experts on ASD issues;

2. Map out the quality of interdisciplinary care of clients with ASD;

3. Learn about the satisfaction of parents having children with ASD with awareness of ASD issues;

4. Learn about the satisfaction of parents having children with ASD with the provided expert assistance.

\section{Questionnaire research in the Olomouc region}

The questionnaire research in the Olomouc region addressed three target groups (speech therapists, experts - specialists in education and special education, psychologists, psychiatrists, paediatricians and neurologists, and parents of children with ASD). At first, we decided to distribute the questionnaires via electronic mail. However, due to a very low return rate 9 questionnaires from speech therapists, 24 questionnaires from experts and 5 questionnaires from parents, we switched to telephone and personal contact. The result was 19 returned questionnaires from speech therapists, 61 from experts and 17 from parents of children with ASD. 
When distributing the questionnaires we were often faced with a negative attitude or denial to the co-operation. Despite our subsequent offer to provide a handbook for parents to the respective experts, the responses were not positive.

Table 1: Number of distributed and returned questionnaires for speech therapists (CR)

\begin{tabular}{|c|c|c|}
\hline $\begin{array}{c}\text { Number } \\
\text { of distributed questionnaires }\end{array}$ & $\begin{array}{c}\text { Number } \\
\text { of returned questionnaires }\end{array}$ & Return rate in \% \\
\hline 41 & 19 & $\mathbf{4 6 . 3 4} \%$ \\
\hline
\end{tabular}

Table 2: Number of distributed and returned questionnaires for experts (CR)

\begin{tabular}{|c|c|c|}
\hline $\begin{array}{c}\text { Number } \\
\text { of distributed questionnaires }\end{array}$ & $\begin{array}{c}\text { Number } \\
\text { of returned questionnaires }\end{array}$ & Return rate in \% \\
\hline 162 & 61 & $\mathbf{3 7 . 6 6 \%}$ \\
\hline
\end{tabular}

Table 3: Number of distributed and returned questionnaires for parents (CR)

\begin{tabular}{|c|c|c|}
\hline $\begin{array}{c}\text { Number } \\
\text { of distributed questionnaires }\end{array}$ & $\begin{array}{c}\text { Number } \\
\text { of returned questionnaires }\end{array}$ & Return rate in \% \\
\hline 28 & 17 & $\mathbf{6 0 . 7 1 \%}$ \\
\hline
\end{tabular}

\section{Analysis of the questionnaire research carried out with speech therapists (Olomouc region)}

Out of the total number of distributed questionnaires (41), 19 were returned, that is $46.34 \%$. The prevailing group of speech therapists who returned the questionnaire concerned therapists working in healthcare (11), and those in practice for more than 15 years ( 8 experts) and between 5 to 10 years (6 experts).

Two items in the questionnaires focused on learning about the basic knowledge regarding autistic spectrum disorders. As sufficient we considered the responses containing a definition somewhat similar to the following: 
Autistic spectrum disorders are one of the most serious disorders of a child's mental development. It is a neurodevelopment disorder with a neurobiological basis. This category includes serious developmental disorders originating in early childhood and characterized by a quantitative disorder of social interaction, communication and inclination to stereotype and ritual behaviour. (Thotová, 2006; Hrdlička, 2004; Ŕičan, Krejčírová et al., 2006). The second item focused on enumeration of basic subcategories falling into this diagnostic category pursuant to International Classification of Diseases 10 (2006) Autistic spectrum disorders. As sufficient we regarded the description of at least 3 basic nosologic units - children's autism, atypical autism and the Asperger syndrome. The results were as follows: There were 10 responses with sufficient basic knowledge about ASD compared to 7 insufficient responses. Therefore, we see a slight prevalence of basic awareness over insufficient awareness. However, the results for the second item are worse. Insufficient knowledge of the basic categories (11) prevails over sufficient knowledge (6).

The importance of speech therapy provided to individuals suffering from autistic spectrum disorder was confirmed by 17 respondents. Its substantiation was correctly explained: "the connection with the triad of specific symptoms, the need to establish a functional communication system enabling socialization, ...” 6 respondents correctly identified the pragmatic linguistic aspects with severe deficit. Three respondents provided an incorrect answer pointing out the phonetic and phonological linguistic aspects.

Therefore, we can say that the addressed speech therapists showed a prevailing correct attitude to the necessity of speech therapy intervention and to the reasons of its realization. However, it is important to emphasize that the questionnaire asked only about basic awareness and, considering the research method, we may not take the received information as definitely decisive.

Subsequent items focused on getting the number of clients with ASD seeing the addressed speech therapists. 14 out of 19 speech therapists confirmed that they have had individuals with ASD in their care. This fact reflects the increasing prevalence of individuals with this disorder (see Hrdlička 2010), which represents another reason to increase awareness and provide correct information about this issue. The answer $0-5$ clients was the most frequent one (8), more than 15 clients had the least frequent answer (1). The most frequently mentioned diagnosis concerned children's autism (13) with obvious disturbance of communication ability and then atypical autism (11). 
The questionnaire also included items related to alternative and augmentative communication (AAC). The reason was a vague, often disapproving attitude to its application. The results, however, were to the contrary -9 speech therapists apply the AAC method. They prefer, in particular, pictograms (10), photographs and objects (9). Satisfaction with the respective communication system is represented, in the majority of cases, with the value of 3 (medium) on the Likert-type scale.

The next section of the questionnaire was aimed at mapping out the situation in mutual co-operation, satisfaction with the provided information and preference to the applied intervention method. The results of the research are as follows: If they had any questions or need help with a client with ASD the majority (16) would contact a psychologist; 8 would contact a specialist in special education and 1 (the lowest frequency) would contact a paediatrician. This result may not be seen as good. Paediatricians should be well informed on the respective issue, should increase awareness and point out the first possible symptoms signalling a risk of ASD. The existing situation is, however, different. More information is, for instance, given in the parents' responses. The item showing that speech therapists have to look up the necessary information themselves (via self-learning) is high (16). It is also worth mentioning that 11 respondents received information while studying at a university, which may be regarded positively. On the Likert-type scale, satisfaction with a degree of awareness at value 3 (medium) prevailed (10). As the most difficult challenge in speech therapy intervention the speech therapists considered the establishment of a communication system (6), managing aggressive behaviour (4) and making contact with the individual with ASD (3). 10 speech therapists mention that they co-operate with a specialist in special education under the interdisciplinary model. The frequency for other experts is very low (2 - paediatrician, neurologist, and psychiatrist). Once again, satisfaction with the interdisciplinary co-operation prevails (5) around value 3 (medium) on the Likert-type scale.

For our internal needs we also included an item asking about attitude to the methodology under preparation. 18 respondents expressed the necessity to establish some methodology. Only 1 said to the contrary. 14 respondents expressed their interest. This result emphasizes the importance of establishing a methodology providing assistance to speech therapists with speech therapy intervention in clients with ASD. 
One of the last items in the questionnaire included a question asking about the attitude of speech therapists to greater awareness and training in the respective area. 11 respondents agree that higher awareness and more extensive training of speech therapists are needed. However, 8 respondents say otherwise. We were surprised by this result ( 8 speech therapists do not require higher awareness or more training!). We think that awareness and continuous training is an absolute necessity in clinical practice of speech therapists. In particular, with respect to the given topic we regard it as very important as ASD has not been fully described and domestic and international research keeps bringing new information.

\section{Analysis of the questionnaire research carried out with experts (Olomouc region)}

Out of the total number of distributed questionnaires - 162, 61 were returned, which is $37.66 \%$. Among the experts who filled in our questionnaire prevailed specialists in special education (17), psychologists (13) and specialists in pre-school education (11). The lowest frequency was in the category others - there was one questionnaire filled in by a social worker. 27 experts have been practicing for more than 15 years, 14 of them for a period from 0 to 5 years and the lowest number for a period from 5 to 10 years.

Two items in the questionnaires focused on learning about the basic knowledge regarding autistic spectrum disorders. As sufficient we considered the responses (to item 4) containing a definition somewhat similar to the following: Autistic spectrum disorders are one of the most serious disorders of a child's mental development. It is a neurodevelopment disorder with a neurobiological basis. This category includes serious developmental disorders originating in early childhood and characterized by a quantitative disorder of social interaction, communication and an inclination towards stereotype and ritual behaviour. (Thotová, 2006; Hrdlička, 2004; Řičan, Krejčírová et al., 2006). The second item (No. 5) focused on enumeration of the basic subcategories falling into this diagnostic category pursuant to International Classification of Diseases 10 (2006) Autistic spectrum disorders. As sufficient we regarded the description of at least 3 basic nosologic units - children's autism, atypical autism and the Asperger syndrome.

The results were as follows: The basic knowledge of ASD issues was regarded as sufficient for 32 respondents, i. e. for more than half of the re- 
turned questionnaires. Insufficient knowledge was seen in 27 respondents. In addition, there are very good results for the second item (No. 5) where sufficient knowledge prevails over insufficient by a proportion of 30:27. The questionnaire, however, asked only about basic awareness and regarding the selected research method the received information may not be considered definitely decisive.

Other items intended to map out the ASD client population with the addressed respondents. 36 of them have been taking care of an individual suffering from ASD and 24 of them did not confirm this fact. This shows that more than half of the experts had or have had some experience in this area. The majority of them have had 0 to 5 clients (19) and only 3 have had 10 to 15 clients. Most often the experts dealt with individuals with children's autism (28), followed by individuals suffering from the Asperger syndrome (19) and the least numerous group suffered from Rett syndrome (3).

The fundamental section of the questionnaires focuses on describing the existing situation in co-operation between the experts and on satisfaction with information provided about ASD. We were also interested in the preferred intervention method and the satisfaction with it. If any clarification or assistance with ASD client was needed, the majority (37) would contact a specialist in special education; 34 respondents would contact a psychologist and 29 would contact a psychiatrist. Only 9 respondents would contact a paediatrician, which makes us assume similar results as for analysing the results of responses given by the speech therapists. The item showing that experts have to look up the necessary information themselves (via self-learning) is high (43). 35 respondents mentioned that they received information about ASD while studying at a university, which may be regarded positively. Satisfaction with the received information was measured using the Likert-type scale (complete dissatisfaction 12345 complete satisfaction). Most frequently the value 3 (medium) appeared - 20 respondents. However, it is important to also mention value 2, which was selected 17 times, and value 1 selected 12 times. This fact points out that the experts are not very satisfied with the information they get. In our opinion that is why 53 respondents spoke of higher awareness as necessary. In the questionnaires the experts say that they most frequently co-operate with a specialist in special education (32), then with a psychologist (24) and psychiatrist (23). The least number of them co-operates with paediatricians (7). This result is alarming and points to the 
need for finding a solution. Satisfaction with interdisciplinary co-operation is shown on the Likert-type scale. The medium value 3 prevails with 18 respondents choosing it. Value 1 (completely dissatisfied) is very alarming and it was confirmed by 15 experts. 25 respondents mentioned that they co-operate with an organization, association, SPC (Special Pedagogic Centre) or other institution specializing in individuals with autistic spectrum disorder; 30 experts replied that they do not co-operate at all.

As the most difficult when working with ASD clients the experts mentioned establishing contact (19), overcoming obstacles in communication (11) and subsequent establishment of a functional communication system (10). This only confirms that autistic spectrum disorders are primarily disorders of communication and early and efficient speech therapy may play an important role and significantly affect the overall development of an individual suffering from ASD.

Furthermore, the research was to provide an answer to what intervention methods are applied by the experts when working with ASD clients and how satisfied the experts are with these methods. The preference of no special intervention method concerned the most frequent result (26), then the TEACCH programme (15) and AAK (9). The reason for absenting special intervention method lies in the fact that the majority of addressed experts (psychologist, psychiatrist, neurologist, paediatricians) do not carry out any direct educational activity requiring the application of any of the methods or approaches above. Based on their answers, the most efficient, and in the Czech Republic, the most frequently used, is the TEACCH method (9).

54 respondents would see as beneficial a handbook that would be the side-product of this research and 55 see it as necessary.

\section{Analysis of the questionnaire research carried out with parents of children with ASD (Olomouc region)}

Out of the total number of distributed questionnaires - 28, 17 were returned, which is $60.71 \%$. Children with ASD whose parents filled in our questionnaire were 3 to 7 years of age (13) and 0 to 3 years of age (3). In terms of diagnostics, the majority concerned children with children's autism (8), the Asperger syndrome (3) and hyperactive disorder combined with mental retardation and stereotype movements (3). The parents noticed the first symptoms between the ages of 2 to 2.5 years old ( 7 cases), between 
the ages of 1.5 to 2 years old ( 4 cases) and one parent even before the child was one year old. In their child's development the parents were specifically disturbed with the late development of speech and specifics in speaking (14), then with eye contact difficulties (10) and problems when playing (9). On the other hand, the parents were disturbed the least with the variations in muscle tension (3). In the majority of cases (13) the children attend a special preschool and currently they are in the care of a specialist in special education (11), speech therapist (8), psychologist (7) and neurologist (7).

Our questionnaire focused primarily on the diagnostic age, satisfaction with the approach of experts and their further recommendations. Most frequently the parents shared their discontentment with a paediatrician (11), then with a psychologist (10) or speech therapist (7). 12 parents were not satisfied with the approach of the respective expert. Only 5 parents expressed satisfaction which is a very negative and an alarming result. The reason for the dissatisfaction stems from late diagnosis(4), lack of provided information (3), insufficient knowledge of the issue (2) and impatience (2). The age of first diagnostic examination was most frequently (6) between the ages of 2 and 2.5 years old and in 4 cases between the ages of 3 and 3.5 years old. In 6 children the final diagnosis was determined between the age of 3 and 3.5 years old and in 4 children between the age of 4 and 4.5 years old. The results revealed that ASD was most frequently diagnosed by a psychiatrist (9), psychologist (5) and neurologist (3). Most often the disorder was diagnosed in Brno. The most frequent remedial recommendations after diagnosing the disorder concerned medication (7), patience and belief in progress (5), and individual learning of the technical literature (5). We may say that such recommendations and steps are not the best after diagnosing ASD.

The questionnaire research carried out among the parents having children with ASD is primarily focused on determining the awareness and satisfaction with the received information. 10 parents mention that they had to look up the information on this problem on their own. 4 respondents were given information by a specialist in special education, 3 by a psychiatrist and only 1 by a paediatrician. This clearly shows that parents are forced to look up information on their own and provision of information by the respective expert is not a matter of course. This finding is also reflected in satisfaction with this area where, based on the Likert-type scale, the value 1 prevails complete dissatisfaction (6). 
In the following section of the questionnaire we focused on determining satisfaction with the educational, psychological and medical assistance. Satisfaction with educational intervention expressed on the Likert-type scale achieved the value 3 - medium. The most frequently given reason for dissatisfaction concerned insufficient knowledge of ASD issues. The evaluation of satisfaction with special educational intervention was very positive. 8 parents selected the value 5 - complete satisfaction and they emphasize individual approach (8), co-operation (7) and good preparation for further education (6). Speech therapy intervention is most often mentioned with a value 1 - complete dissatisfaction (5). On the other hand we also received an antagonist value 5 - complete satisfaction (3). The reason for dissatisfaction lies again in insufficient knowledge of the issue. For psychological interventions parents selected a value of 2 in 5 cases and a value of 3 in 4 cases. Therefore, there is an inclination to dissatisfaction with the provided psychological care. The reasons given include diagnostics without follow-up care (8), lack of experience with ASD (5), no interest in a client and addressing his/her problems (2) and even arrogance of the expert (1). Similar results were received for medical care. The reasons include long waiting time at the doctor's before examination (4), lack of awareness (3) and short time spent examining the child (2).

8 respondents take advantage of counselling services, primarily SPC (8) and other non-profit organizations (4). Parents most frequently use special educational assistance (7). Dissatisfaction once again results from lack of awareness and limited experience of experts with ASD. Upon providing services to parents, there is a lack of awareness (9), empathy and trust (6), humane as well as professional attitude (4) and legal counselling (3).

In conclusion we cite some of the parents' statements. "After diagnosis it was recommended - if you need to know anything, come and ask (perfect advice for parents who do not know what autism really is)." "The most common attitude at paediatric neurology, EEG in Olomouc: Despite an appointment for a specific hour, waiting time of about 2 hours and no way to enable the child to become familiar with the environment. Basically as soon as we opened the door, the nurse was putting the "cap" on my daughter's head and was surprised she did not like it - results: a child impossible to be examined." "SPC does not work as I think it should. The parent needs to find the information on his own, likewise the method on how to work with the 
child." "SPC staff did not have time over the period of two and half years to see my son in pre-school. They assess and conclude based on seeing him in their office once a year." "When addressing problems we were told to start our son on medication." "The paediatrician has not been able to learn what autism is and thus the attitude to our son is not good."

\section{Conclusion of the questionnaire research in the Olomouc region}

A questionnaire research aimed at mapping the current situation in care provided in early care and speech therapy intervention in individuals with autistic spectrum disorder was carried out in the Olomouc region between April and July 2010. We prepared three types of questionnaires - for speech therapists, experts (specialists in education and in special education, psychologists, psychiatrists, paediatricians and neurologists) and parents of children with autistic spectrum disorder. After the initial electronic distribution of questionnaires we switched to telephone and personal contact with the respondents due to low return rate. The results brought 19 questionnaires filled out by speech therapists, 61 by other experts and 17 by parents with ASD children.

At this point we compare the principal sections of the questionnaires for speech therapists, experts and parents of ASD children. As the key areas in the questionnaires for experts (speech therapists, specialists in education and special education, psychologists, psychiatrists, paediatricians and neurologists) we defined the following:

1. Basic knowledge of ASD;

2. Number of clients with ASD;

3. Co-operation with other experts when addressing issues with ASD;

4. Satisfaction with information and possible need of further education and training.

In questionnaires for parents with ASD children we decided to focus on two fundamental areas:

1. Satisfaction with the attitude of experts;

2. Satisfaction with the received information and awareness.

To find out the basic awareness to ASD is seen as very important. Therefore, in both the questionnaires for speech therapists and experts there are two items mapping out the basic awareness on this area. After analysing the 
responses from speech therapists and experts we may summarize that the knowledge of basic ASD issues and awareness of individual nosologic units under the diagnostic category "Autistic spectrum disorders" is at a medium level with a moderate predominance of sufficient knowledge over insufficient knowledge. We are, of course, aware that the results are, with respect to the research method, only of informational value.

The questionnaires were also designed to receive an answer on the number of ASD clients with the respective experts. We regard these results very important since, together with the awareness of the experts of this issue and satisfaction of parents with the provided care, they point out the topicality of this area. 14 speech therapists out of 19 and 36 experts out of 61 state that they have had or they have clients with ASD in their care. This shows that $62.5 \%$ of all experts have achieved certain experience with this disorder. Considering other factors such as dissatisfaction with the available information and the provided care (see below) we think it is necessary to focus more on educating the respective experts.

We consider co-operation with experts when addressing specific problems of ASD clients to be absolutely essential. This actual situation is described in the following result. Most often all the addressed experts co-operate with a specialist in special education. Out of the other professions (paediatrician, psychologist, neurologist, and psychiatrist) speech therapists, in particular, co-operate the least. Analyzing the questionnaires filled out by the experts, the results are better. 24 respondents mentioned co-operation with a psychologist. As very alarming we consider the minimal degree of co-operation with a paediatrician. This applies both to responses given by the speech therapists (2) and other experts (7). In our opinion, the role of a paediatrician is very important as they map out early development of a child. Their sufficient education may result in early detection of the first warning symptoms enabling, after subsequent special examination (psychological, neurological, auditory and other), early diagnostics, diagnoses and possibly also special educational intervention. The above reasons point out the importance of systemic education and the provision of information about ASD to all respective experts. We regard as important both the enlightenment activities - articles in journals, presentations at conferences here and abroad - and systemic education (courses).

The following responses reflected that the majority of experts are not satisfied with the provided information. On the Likert-type scale the values 
3 to 1 prevail. 17 times the experts selected the value of 2 and 12 times they selected the value of 1 . The majority of them agree that they have to find the information on their own from studying technical literature. On the other hand, it should be stressed out that self-learning and further education should be a matter of course for any profession and for these experts in particular. At present, the offer of educational courses and seminars (e.g. Institute of educational and psychological counselling of the Czech Republic, Apla Brno, Apla Praha, Autistik, etc.) is very variable and adjusted to suit the needs of the respective professions. The question is what kind of incentives these experts would need to get involved in further education.

The overall situation was illustrated by statements of parents having children with ASD. 12 out of 17 parents were not satisfied with the attitude of the respective experts. The main reasons included late diagnosis, lack of provided information, limited knowledge of the field and impatience. 10 out of 17 parents had to find the information themselves, in particular through studying technical literature. Only 4 parents gained information from a specialist in special education, 3 from a psychiatrist and one from a paediatrician. These results are very alarming and only confirm the situation described above. To conclude we cite one parent: "The paediatrician recommended us to sign up our child at a pre-school and that speech would develop among a group of children. And when in a fit of rage, just to shower him with cold water. Upon our request we saw a psychologist. After our son had been diagnosed the paediatrician said that over the 15 years of his practice he had not seen an autistic child."

\section{Questionnaire research in Slovakia (Bratislava)}

The questionnaire research in Slovakia (Bratislava) addressed three target groups (speech therapists, experts - specialists in education and special education, psychologists, psychiatrists, paediatricians and neurologists, and parents of children with ASD). At first, we decided to distribute the questionnaires via electronic mail. However, due to a very low return rate $\mathbf{2}$ questionnaires from speech therapists, 3 questionnaires from experts and 0 questionnaires from parents, we used contacts from Mgr. Lucie Pastieriková and contacted experts via telephone and then electronically mailed the questionnaires again. Despite this effort the return rate remained very low: 7 returned questionnaires from speech therapists, 9 from other experts and 
4 from parents having children with ASD. The tables specifying the number of distributed and returned questionnaires from speech therapists, experts and parents with ASD children are provided below.

The return rate is to be considered as insufficient and the results do not entail any informative value. Therefore, it was not possible to analyse, generalize and compare the results. Visualized results (tables, bar charts) are shown below.

Table 4: Number of distributed and returned questionnaires for speech therapists

\begin{tabular}{|c|c|c|}
\hline $\begin{array}{c}\text { Number } \\
\text { of distributed questionnaires }\end{array}$ & $\begin{array}{c}\text { Number } \\
\text { of returned questionnaires }\end{array}$ & Rate of return in \% \\
\hline 34 & 7 & $\mathbf{2 0 . 5 9} \%$ \\
\hline
\end{tabular}

Table 5: Number of distributed and returned questionnaires for experts

\begin{tabular}{|c|c|c|}
\hline $\begin{array}{c}\text { Number } \\
\text { of distributed questionnaires }\end{array}$ & $\begin{array}{c}\text { Number } \\
\text { of returned questionnaires }\end{array}$ & Rate of return in \% \\
\hline 128 & 9 & $\mathbf{7 . 0 3} \%$ \\
\hline
\end{tabular}

Table 6: Number of distributed and returned questionnaires for parents

\begin{tabular}{|c|c|c|}
\hline $\begin{array}{c}\text { Number } \\
\text { of distributed questionnaires }\end{array}$ & $\begin{array}{c}\text { Number } \\
\text { of returned questionnaires }\end{array}$ & Rate of return in \% \\
\hline 13 & 4 & $\mathbf{3 0 . 7 7 \%}$ \\
\hline
\end{tabular}

\section{Conclusion}

The research called Analysis of the current situation concerning care provided in early age and speech therapy intervention in individuals with autism spectrum disorder was carried out in the Olomouc region and abroad (Slovak Republic - Bratislava) between April and June 2010. The return rate of questionnaires in the Olomouc region was $\mathbf{4 1 . 9 9 \%}$ out of all questionnaires and abroad (Slovak Republic - Bratislava) it was $\mathbf{1 1 . 4 3 \%}$. Owing to an insuf- 
ficient rate of return from abroad, we may not use the received information. Therefore, we only carried out the analysis and evaluation in the Olomouc region where the rate of return may be considered relevant with respect to the subsequent generalization.

The objectives of the research we set in the introduction may thus be considered, with respect to the insufficient rate of return, as just partially fulfilled.

The data received through questionnaires distributed in the Olomouc region are considered as very valuable. The summary of the fundamental resulting information is given in the chapter Conclusion of the questionnaire research in the Olomouc region.

The research pointed out serious problems, emphasized areas that need to be developed and outlined possible solutions. Further research and actions towards higher awareness, sufficient professional soundness and the highest possible satisfaction of parents and children with ASD is regarded as important.

\section{Bibliography}

ČADILOVÁ, V., JŮN, H., THOROVÁ, K. A KOL. Agrese u lidí s mentální retardací a s autismem. Praha: Portál, 2007. ISBN 978-80-7367-319-2.

JELÍNKOVÁ, M. Autismus I. Problémy komunikace dètí s autismem. Praha: Institut pedagogicko-psychologického poradenství ČR, 1999.

KLENKOVÁ, J. Logopedie. Praha: Grada, 2008. ISBN 80-247-1110-9.

KURT-LASOCKA. Z problematyki autyzmu. Wroclaw: Uniwersytet Wroclawski, 1994. ISBN 83-229-1046-0.

MEZINÁRODNÍ KLASIFIKACE NEMOCÍ - 10. REVIZE. Duševní poruchy a poruchy chování. Popis klinických príznaků a diagnostická vodítka. Praha: Psychiatrické centrum, 2006. ISBN 80-85121-11-5.

PEETERS, T. Autismus od teorie k výchovně- vzdèlávací intervenci. Praha: Scientia, 1998. ISBN 80-7183-114-X.

ŘÍČAN, P., KREJČĆǏROVÁ, D. Dětská klinická psychologie. Praha: Grada, 2006. ISBN 80-247-1049-8.

WEBER, D. Der Frühkindliche Autismus. Bern, 1970. 
Was ist autistische Störung? Diagnostik. Therapie und Frühförderung...

[2009-12-9] Available at: http://www2.autismus.de/pages/startseite.php Was ist autismus? Checklisten zur Früherkennung... [2009-12-9]

Available at: http://www.autismus-online.de/autismus.htm

\section{Contact}

Mgr. Alena Ríhová

Institute of Special Education, Faculty of Education, Palacky University, Olomouc

Žižkovo nám. 5, 77140 Olomouc

e-mail: alena.rihova@email.cz

tel.: 585635321 